\title{
Security-Oriented Polar Coding Based on Channel-Gain-Mapped Frozen Bits
}

\author{
Yuli Yang, Senior Member, IEEE, and Wenyao Li
}

\begin{abstract}
In this paper, a novel design named securityoriented polar coding (SOPC) is proposed to enhance the physical layer security (PLS), where the active pattern of frozen bits in a transmission is determined by random channel gain of the legitimate link. Since the channel gain value is not exchanged between the legitimate transmitter and the desired receiver, eavesdroppers cannot ascertain the frozen bit pattern engaged in the legitimate polar coding. When the signal-to-noise ratio (SNR) is low, eavesdroppers are unable to appropriately decode the confidential information delivered over the legitimate link. As the SNR increases, eavesdroppers may have chance to sort out the correct frozen bit pattern through a brute-force search. However, this chance is significantly reduced by our SOPC. We design the SOPC for both single-input-single-output singleantenna eavesdropper (SISOSE) and multiple-input-multipleoutput multi-antenna eavesdropper (MIMOME) channels. Its PLS functioning is assessed in terms of the error rate difference between the legitimate receiver and the eavesdropper. Illustrative simulation results substantiate that the SOPC design guarantees degraded decoding performance at eavesdroppers, for both SISOSE and MIMOME channels, even in the presence of a powerful eavesdropper possessing infinite computational resources.
\end{abstract}

Index Terms - Physical layer security (PLS), security-oriented polar coding (SOPC), frozen bits, channel gain, time division duplexing (TDD).

\section{INTRODUCTION}

The confidentiality and authenticity of communications rely on the upper-layer secret key exchange in traditional network security, which requires extra resources and reduces effective throughput [1]. To address the challenges of high-dynamic infrastructure for exponentially increasing data services and massive connectivity in emerging and future wireless networks, the concept of physical layer security (PLS) has been proposed as a potential solution to secret key generation [2].

The underlying idea of PLS approaches is the link signature, which refers to the inherent randomness of channel characteristics between a pair of legitimate users, exploited for the generation of physical-layer secret key to provide informationtheoretical security. Specifically in time division duplexing (TDD) systems [3], [4], the link signature is nearly reciprocal and the pair of legitimate users under study can generate a secret key from their own estimates of the common channel state information (CSI), without any feedback signalling [5].

This work was supported in part by National Key Research \& Development Program of China under Grant 2018YFB2202202 and National Natural Science Foundation of China under Grant 61771188.

Y. Yang is with the School of Engineering, University of Lincoln, Lincoln LN6 7TS, U.K. (e-mail: yyang@lincoln.ac.uk).

W. Li is with the Department of Electronics, Peking University, Beijing 100871, China (e-mail: liwenyao@stu.pku.edu.cn).
In practice, sounding mechanisms have been supported by diverse mobile communication standards, e.g., IEEE 802.162017 [6] and 5G-NR [7], for estimating the uplink CSI to enable user scheduling and link adaption. Moreover, through accurate calibration of the transceiver radio frequency chains, the channel reciprocity in TDD allows the downlink CSI to be estimated from the sounding over uplink. Since the legitimate CSI is not transmitted, the third parties are prevented from obtaining any knowledge of it. Hence, eavesdroppers cannot gain access to the secret key and, therefore, they will fail to decode the confidential information [8], [9].

To further enhance the information-theoretical security, a number of physical-layer techniques have been developed or renovated, e.g., artificial noise [10] and jamming [11], [12], to degrade the third parties' channels. As intensive cryptographic computations are not required and routine signal processing in the upper-layer secret key exchange is avoided, the PLS is able to improve the resource utilisation efficiency [13]. Besides, the PLS is compatible with the upper-layer encryption, for achieving ultimate security [14], [15].

In the literature, the majority of PLS techniques utilised multi-antenna transmissions to jam eavesdroppers' wiretapping while improving desired receivers' performance, under the assumption that the transmitter knows the CSI of both legitimate link and wiretapping link [16]. In reality, the transmitter can always get the CSI of the link spanning from itself to the desired receiver without any feedback signalling in TDD systems. However, it is impractical to assume the transmitter is capable of getting the wiretapping CSI or its statistics if the eavesdropper is a non-authorised subscriber. In [17], an approach to power allocation was suggested for artificial noise injection when the wiretapping CSI or its statistics is not perfectly known by the transmitter. In [18], we proposed an artificial noise scheme for single-antenna systems over multi-path channels, where the transmitter is not required to know wiretapping CSI at all. In [19], retransmissions were exploited for adaptive artificial noise injection, given that the wiretapping CSI is not available to the legitimate transmitter. Although these works removed the impractical assumption from the PLS implementation, they were still based on the artificial noise design to address this issue, which inevitably caused an increase in the peak-to-average power ratio and sacrificed certain power resources in the interference generation.

Different from the artificial noise or jamming techniques, we have been attempting to address more practical PLS solutions based on the randomness of information sources rather than that of wireless channels. In particular, we take the formatting of information sources as a secret key. In [20], we proposed an 
information-source-based PLS solution to degrade eavesdroppers' detection performance instead of their channel quality, by varying the spatial modulation patterns of the bit-to-symbol mapping and the transmit antenna (TA) selection according to the legitimate channel gain. The strategy is making the spatial modulation pattern known to the desired receiver but unknown to eavesdroppers, which guarantees the former's detection performance and prohibits the latter from extracting the information delivered in the legitimate link. This solution leads to a plainer transmission medium and provides more freedom for the communication protocol design, especially in ad-hoc networks [21] and non-orthogonal multiple access systems [22]. Furthermore, we proposed a security-oriented trellis coded spatial modulation in [23], which not only varies the mapping patterns but also optimises the free Euclidean distance between the constellation points received by the desired receiver, to improve the legitimate link performance while enhancing the PLS.

In this work, we propose a novel design of polar coding for the PLS purpose, referred to as security-oriented polar coding (SOPC), based on the channel-gain-mapped frozen bits. Elaborating slightly further, the active pattern of frozen bits in the SOPC is determined by the instantaneous channel gain of the legitimate link.

Polar codes have been accepted by the $3^{\text {rd }}$ generation partnership project in the $5 \mathrm{G}$ wireless systems standardization process, as channel coding for the uplink and downlink control information within the enhanced mobile broadband communication service [24]. The polar coding is proposed on the recursive coding basis to achieve channel polarisation [25]. It transforms $N$ independent copies of a given binaryinput discrete memoryless channel (B-DMC) into $N$ dependent channels that exhibit various levels of reliability. As $N$ increases, the fraction of the resultant channels having near one capacity approaches the symmetric channel capacity of the given B-DMC, and almost all the rest of the resultant channels have near zero capacity. By virtue of the channel polarisation phenomenon, the most reliable channels are selected to carry information bits at full rate, whilst the frozen bits are delivered through the remaining. The concatenated structure of polar codes prompts a recursive formalisation of low-complexity decoding algorithms, e.g., successive cancellation (SC) [26] and successive cancellation list (SCL) [27]. As the frozen bits are fixed and known to the receiver, they are utilised as guidepost in the polar decoding to direct the correct path, which dramatically boost the decoding accuracy.

The reality that the polar decoding has to rely on the frozen bits is well-conditioned for the security-oriented design. Motivated by this, we vary the patterns of frozen bits engaged in the transmitter's polar coding according to the legitimate channel gain, which is taken as a physical-layer secret key. Since the channel reciprocity in TDD allows the transmitter and desired receiver to observe the same CSI without using feedback signalling, the desired receiver always knows the legitimate channel gain and the instantaneous pattern of frozen bits. This guarantees robust and low-complexity polar decoding at the desired receiver. However, as eavesdroppers are unable to obtain the legitimate channel gain value through wiretapping if the TDD mode is adopted by the legitimate link, they cannot ascertain the frozen bit pattern exploited by the transmitter for the time being. Accordingly, eavesdroppers always fail to carry out the polar decoding in an appropriate manner.

We design the variation of frozen bit patterns for two wiretap scenarios: single-input-single-output single-antenna eavesdropper (SISOSE) and multiple-input-multiple-output multiantenna eavedsdropper (MIMOME) channels. Since the frozen bit patterns, used as secret keys, come from a finite set, our SOPC design does not provide information-theoretical security. Based on the uniform polar coding construction, eavesdroppers can still decode a certain amount of information bits, though at much higher error rates than the legitimate receiver does. To further strengthen the security achieved by the SOPC, artificial noise needs to be sent by the transmitter to fully protect the confidential information. In this case, the SOPC design will contribute to the deduction of the transmit power consumed by the artificial noise, thus improving the energy efficiency.

The novelties and contributions of this work are highlighted below in three aspects.

- PLS solution in SISOSE channels: The frozen bit patterns are varied in line with the instantaneous channel gain of the legitimate link. The legitimate channel gain range is partitioned into several non-overlapping consecutive intervals, and the active pattern of frozen bits is determined by the interval in which the instantaneous channel gain falls. As the polar coding naturally supports rate adaptation, the frozen bit patterns for different channel gain intervals are automatically varied to match the bit-channel reliability in the polar coding construction. Therefore, the transmitter does not need to employ adaptive modulation for achieving the channel capacity. This efficiently inhibits eavesdroppers from detecting the legitimate channel gain level through observing the modulation method used by the transmitter. As eavesdroppers do not know the instantaneous pattern of frozen bits engaged in the transmitter's polar coding, they cannot successfully decode the information conveyed over the legitimate link. Our PLS solution in SISOSE channels not only enhances the security performance of single-antenna wiretap channels, but also breaks the limitation of multiantenna configuration, imposed by most of current PLS techniques, on the transmitter.

- PLS solution in MIMOME channels: The frozen bit pattern in a transmission is determined by the channel gain pattern in the legitimate link of the moment. The legitimate channel gain pattern is defined as the permutation containing the channel gains from all TAs to the desired receiver, in descending or ascending order. Since eavesdroppers are blind to the instantaneous channel gain pattern of the legitimate link, they cannot get access to the frozen bit pattern and hence will fail to successfully decode the information transmitted from the transmitter. In addition to the frozen bit pattern activated as a physicallayer secret key, the transmitter sends out artificial noise to further degrade the wiretapping link quality. As more TAs are embraced for the artificial noise injection, much 
more legitimate channel gain patterns are introduced and the legitimate link security will be fully guaranteed.

- Powerful Eavesdroppers' Decoding: To evaluate the maximum possible information leakage from our SOPC design, we assume that eavesdroppers, with extremely high computing capability, might implement powerful detectors using a brute-force search of all possible frozen bit patterns, for unveiling the information delivered over the legitimate link. As the signal-to-noise ratio (SNR) increases, the chance that eavesdroppers may work out the correct frozen bit pattern through a brute-force search gets higher. Nevertheless, simulation results against this backdrop substantiate that our SOPC design is robust to powerful eavesdroppers possessing unlimited computational resources.

In detailing the above contributions, the remainder of this paper is organized as follows. Section II proposes the fundamental principles of the SOPC and evaluates its PLS performance in SISOSE channels. Section III amalgamates the SOPC with artificial noise to enhance the PLS in MIMOME networks. Finally, this paper is concluded in Section IV.

Notations: Vectors and matrices are denoted by boldface lowercase and uppercase letters, respectively. In particular, $\mathbf{0}_{1 \times M}$ denotes the $1 \times M$ zero vector and $\mathbf{I}_{M}$ denotes the $M \times M$ identity matrix. The conjugate, the transpose, the conjugate transpose, the modulus, and the Frobenius norm operators are denoted by $(\cdot)^{*},(\cdot)^{\mathrm{T}},(\cdot)^{\dagger},|\cdot|$ and $\|\cdot\|_{\mathrm{F}}$, respectively. The cardinality of a set $\mathcal{A}$ is denoted by $\sharp \mathcal{A}$. The decimal-to-binary converter is denoted by $\mathcal{B}(\cdot)$, and $\oplus$ stands for the mod-2 addition. The $M \times N$ complex number domain is denoted by $\mathbb{C}^{M \times N}$, and the least integer function is denoted by $\lceil\cdot\rceil$.

\section{SOPC IN SISOSE CHANNELS}

In this section, the SOPC is proposed for a single-antenna wiretap channel.

\section{A. Channel Model}

Consider a single-antenna wiretap channel as shown in Fig. 1, where the transmitter Alice delivers confidential information to the legitimate receiver Bob. An eavesdropper Eve is wiretapping Alice's transmissions and attempts to extract the confidential information. Herein, Alice, Bob, and Eve are all single-antenna devices.

The legitimate (Alice-Bob) and wiretapping (Alice-Eve) links, denoted by $h$ and $g$, are independent and identically distributed (i.i.d.) block Rayleigh-fading channels. The channel coefficients $h$ and $g$ are drawn from a zero-mean, unit-variance complex Gaussian distribution $\mathcal{C N}(0,1)$ at the beginning of each block and remain constant for $L$ symbols, where $L$ represents the block length. This process is repeated for every block in an i.i.d. fashion.

For an arbitrary block of $L$ symbols $\mathbf{s}=\left[s_{1}, s_{2}, \cdots, s_{L}\right]$ transmitted from Alice, the signals received at Bob and Eve, denoted by the $1 \times L$ vectors $\mathbf{y}_{\mathrm{B}}$ and $\mathbf{y}_{\mathrm{E}}$, are expressed as

$$
\mathbf{y}_{\mathrm{B}}=h \mathbf{s}+\mathbf{z}_{\mathrm{B}}
$$

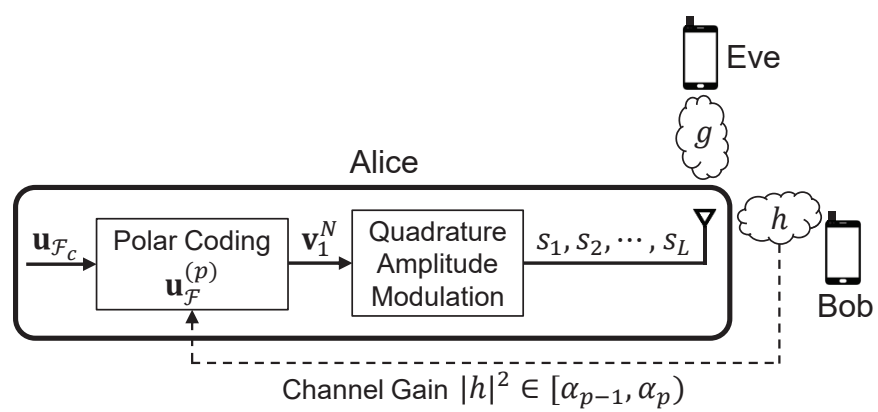

Fig. 1. The SOPC in a single-antenna wiretap channel.

and

$$
\mathbf{y}_{\mathrm{E}}=g \mathbf{s}+\mathbf{z}_{\mathrm{E}},
$$

respectively, where the $1 \times L$ vectors $\mathbf{z}_{\mathrm{B}}$ and $\mathbf{z}_{\mathrm{E}}$, obeying the distribution $\mathcal{C N}\left(\mathbf{0}_{1 \times L}, \sigma_{Z}^{2} \mathbf{I}_{L}\right)$, contain Bob's and Eve's additive white Gaussian noise (AWGN) components, respectively.

\section{B. Polar Code Design}

For a given polar-coded transmission at Alice, a sequence composed of $K$ information bits is encoded into a sequence composed of $N$ coded bits, using $N-K$ frozen bits. The polar coding is characterised by its parameters $\left(N, K, \mathcal{F}, \mathbf{u}_{\mathcal{F}}\right)$, where the set $\mathcal{F} \subset\{1,2, \cdots, N\}$, having cardinality $\sharp \mathcal{F}=$ $N-K$, and the $1 \times(N-K)$ vector $\mathbf{u}_{\mathcal{F}}$ specify the location and the pattern of the $N-K$ frozen bits, respectively. The information bit sequence is denoted by a $1 \times K$ vector $\mathbf{u}_{\mathcal{F}_{c}}$, where the set $\mathcal{F}_{c}$, having cardinality $\sharp \mathcal{F}_{c}=K$, is the complement of $\mathcal{F}$ and determines the location of $K$ information bits. The coded bit sequence is denoted by a $1 \times N$ vector $\mathbf{v}_{1}^{N}$.

The wireless channel reciprocity in the TDD mode allows Alice and Bob to observe the same coefficient $h$. As no feedback signalling is used to deliver the channel coefficient, Eve cannot obtain any information on $h$ through wiretapping. Therefore, we exploit the random channel gain $|h|^{2}$ to determine the pattern of frozen bits for polar code design, which can be deemed as a secret key for the PLS enhancement.

The total channel gain range $[0,+\infty)$ is partitioned into $P$ non-overlapping consecutive intervals $\left[\alpha_{p-1}, \alpha_{p}\right), p=$ $1,2, \cdots, P$, with $\alpha_{0}=0$ and $\alpha_{P}=+\infty$. To ensure that the channel gain $|h|^{2}$ falls in each interval $\left[\alpha_{p-1}, \alpha_{p}\right)$ at the same probability $1 / P$, the boundary points are set according to

$$
\begin{array}{r}
\int_{\alpha_{p-1}}^{\alpha_{p}} e^{-x} d x=e^{-\alpha_{p-1}}-e^{-\alpha_{p}}=1 / P, \\
p=1,2, \cdots, P,
\end{array}
$$

where the probability density function (pdf) of the channel gain $|h|^{2}$ is $f_{X}(x)=e^{-x}$.

There are $P$ candidate patterns of frozen bits at Alice, denoted by $\mathbf{u}_{\mathcal{F}}^{(1)}, \mathbf{u}_{\mathcal{F}}^{(2)}, \cdots, \mathbf{u}_{\mathcal{F}}^{(P)}$. When the channel gain $|h|^{2}$ falls in the $p^{\text {th }}$ interval $\left[\alpha_{p-1}, \alpha_{p}\right)$, Alice chooses the $p^{\text {th }}$ pattern of frozen bits, $\mathbf{u}_{\mathcal{F}}^{(p)}$, for the polar coding. Note that, Alice does not employs adaptive modulation. The same quadrature amplitude modulation (QAM) method is applied to all the 
$P$ channel gain intervals, for fear that Eve could determine the legitimate channel gain level through observing which QAM method is being used in the legitimate link. As such, the polar code in each interval is constructed to match the reliability calculated using the Bhattacharyya parameter [25], for achieving the block Rayleigh-fading channel capacity of the moment, i.e., $\log _{2}\left(1+|h|^{2} E_{s} / \sigma_{Z}^{2}\right)$ bits per channel use, where $E_{s}$ is the energy per QAM symbol in joules.

As polar coding is formulated as a practical scheme for boosting the channel cutoff rate, which governs the pairwise error probability in decoding, to the channel capacity [28], the $A$-ary QAM using a polar code of rate $K /\left(N \log _{2} A\right)$ achieves almost the same error probability for $A=2,4,8, \cdots$, at the same data rate $K / N$, specifically given low SNRs. Consequently, although adaptive modulation is not employed, the adaptive polar code rate guarantees the maximum achievable data rate in each channel gain interval. Thanks to the elegant construction of polar coding, both the security and the adaptivity in communications are addressed by our SOPC design. More specifically, the $p^{\text {th }}$ pattern $\mathbf{u}_{\mathcal{F}}^{(p)}$ contains $N-K_{p}$ frozen bits, to achieve the polar code rate $K_{p} / N$ in the interval $\left[\alpha_{p-1}, \alpha_{p}\right), p=1,2, \cdots, P$. For example, the $p^{\text {th }}$ candidate pattern of frozen bits can be designed as

$$
\mathbf{u}_{\mathcal{F}}^{(p)}=\mathcal{B}\left(N-K_{p}\right)
$$

where $\mathcal{B}(\cdot)$ denotes the decimal-to-binary converter.

Without loss of generality, Alice's encoding process in an arbitrary transmission is expressed as

$$
\mathbf{v}_{1}^{N}=\mathbf{u}_{\mathcal{F}_{c}} \mathbf{E}_{N}\left(\mathcal{F}_{c}\right) \oplus \mathbf{u}_{\mathcal{F}}^{(p)} \mathbf{E}_{N}(\mathcal{F}),
$$

given that $|h|^{2} \in\left[\alpha_{p-1}, \alpha_{p}\right)$, where the $N \times N$ polar encoding matrix $\mathbf{E}_{N}=\mathbf{E}_{2}^{\otimes \log _{2} N}$ is the $\left(\log _{2} N\right)^{\text {th }}$ Kronecker product of the kernel matrix $\mathbf{E}_{2}=\left[\begin{array}{ll}1 & 0 \\ 1 & 1\end{array}\right]$. The submatrix $\mathbf{E}_{N}\left(\mathcal{F}_{c}\right)$, containing the rows with indices in the set $\mathcal{F}_{c}$, deals with the information bits $\mathbf{u}_{\mathcal{F}_{c}}$. The submatrix $\mathbf{E}_{N}(\mathcal{F})$, containing the rows with indices in the set $\mathcal{F}$, deals with the frozen bits $\mathbf{u}_{\mathcal{F}}^{(p)}$.

Then, the polar coded bits $\mathbf{v}_{1}^{N}$ are modulated through $A$-ary QAM, yielding $L=N / \log _{2} A$ symbols $\mathbf{s}=\left[s_{1}, s_{2}, \cdots, s_{L}\right]$ to be transmitted from Alice. Since Eve is unable to obtain any information on the random channel gain $|h|^{2}$ of the legitimate link, she has no knowledge on the pattern of frozen bits adopted by Alice. Hence, Eve does not have a correct basis to successfully decode Alice's polar coding in a normal way. This will enhance the confidentiality of the information conveyed over the legitimate link.

\section{Polar Decoding}

Herein, the polar decoders utilised by Bob and Eve are presented, under the assumption that the legitimate CSI $h$ is perfectly known at Bob and the wiretapping CSI $g$ is perfectly known at Eve.

1) Bob's Decoding: Thanks to the channel reciprocity in the TDD mode, Bob always knows the instantaneous channel gain $|h|^{2}$ of each block in the legitimate link. Accordingly, the pattern of frozen bits, $\mathbf{u}_{\mathcal{F}}^{(p)}$, exploited by Alice is also known to Bob. Upon receiving the signals $\mathbf{y}_{\mathrm{B}}$ given by (1), Bob can

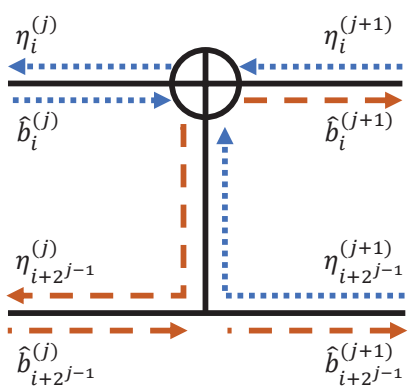

Fig. 2. The SC decoding process for the mod-2 sum of the $i^{\text {th }}$ and the $\left(i+2^{j-1}\right)^{\text {th }}$ bits at the $j^{\text {th }}$ level.

decode the information bits $\mathbf{u}_{\mathcal{F}_{c}}$ using popular polar decoding algorithms, e.g., SC [26], in a normal way.

For an arbitrary polar-coded bit sequence $\mathbf{v}_{1}^{N}=$ $\left[v_{1}, v_{2}, \cdots, v_{N}\right]$, Bob calculates the log-likelihood ratio (LLR) of the $i^{\text {th }}$ bit $v_{i}$, using

$$
L L R_{\mathrm{B}}\left(v_{i}\right)=\ln \frac{\sum_{v_{i}=0} \exp \left(-\frac{\left|y_{\mathrm{B}}\left(v_{i}\right)-h s\left(v_{i}\right)\right|^{2}}{\sigma_{Z}^{2}}\right)}{\sum_{v_{i}=1} \exp \left(-\frac{\left|y_{\mathrm{B}}\left(v_{i}\right)-h s\left(v_{i}\right)\right|^{2}}{\sigma_{Z}^{2}}\right)},
$$

where $y_{\mathrm{B}}\left(v_{i}\right)$ and $s\left(v_{i}\right)$ represent Bob's received and Alice's transmitted signals that contain the bit $v_{i}, i=1,2, \cdots, N$.

With the aid of these obtained LLRs, Bob's SC decoding of polar codes is illustrated in Fig. 2, where the operation is carried out for the mod-2 sum of the $i^{\text {th }}$ and the $\left(i+2^{j-1}\right)^{\text {th }}$ bits at the $j^{\text {th }}$ level, $b_{i}^{(j)}$ and $b_{i+2^{j-1}}^{(j)}, i=1,2, \cdots, N$, $j=1,2, \cdots, \log _{2} N$. The LLR and the estimation of $b_{i}^{(j)}$ is denoted by $\eta_{i}^{(j)}$ and $\hat{b}_{i}^{(j)}$, respectively. In particular, $\eta_{i}^{\left(\log _{2} N+1\right)}=L L R_{\mathrm{B}}\left(v_{i}\right)$ and $b_{i}^{(1)}=u_{i}$. We remark that, $u_{i}$ is a frozen bit in $\mathbf{u}_{\mathcal{F}}^{(p)}$ if $i \in \mathcal{F}$ and an information bit in $\mathbf{u}_{\mathcal{F}_{c}}$ if $i \in \mathcal{F}_{c}$.

Firstly, the LLR $\eta_{i}^{(j)}$ is calculated using [29]

$$
\begin{aligned}
\eta_{i}^{(j)}= & \operatorname{sign}\left(\eta_{i}^{(j+1)}\right) \operatorname{sign}\left(\eta_{i+2^{j-1}}^{(j+1)}\right) \\
& \times \min \left(\left|\eta_{i}^{(j+1)}\right|,\left|\eta_{i+2^{j-1}}^{(j+1)}\right|\right) .
\end{aligned}
$$

If the bit $b_{i}^{(j)}$ is determined by the frozen bit(s) in $\mathbf{u}_{\mathcal{F}}^{(p)}$, Bob will have $\hat{b}_{i}^{(j)}=b_{i}^{(j)}$, which is know to him; otherwise, the estimation of $b_{i}^{(j)}$ is based on the obtained LLR $\eta_{i}^{(j)}$, i.e.,

$$
\hat{b}_{i}^{(j)}= \begin{cases}0, & \eta_{i}^{(j)}>0 ; \\ 1, & \eta_{i}^{(j)}<0 .\end{cases}
$$

Subsequently, the LLR $\eta_{i+2^{j-1}}^{(j)}$ is calculated by

$$
\eta_{i+2^{j-1}}^{(j)}=(-1)^{\hat{b}_{i}^{(j)}} \eta_{i}^{(j+1)}+\eta_{i+2^{j-1}}^{(j+1)},
$$

based on which $\hat{b}_{i+2^{j-1}}^{(j)}$ is estimated if the bit $b_{i+2^{j-1}}^{(j)}$ is not generated by the frozen bit(s) in $\mathbf{u}_{\mathcal{F}}^{(p)}$.

Then, Bob estimates the bits $b_{i}^{(j+1)}$ and $b_{i+2^{j-1}}^{(j+1)}$ as

$$
\begin{aligned}
\hat{b}_{i}^{(j+1)} & =\hat{b}_{i}^{(j)} \oplus \hat{b}_{i+2^{j-1}}^{(j)}, \\
\hat{b}_{i+2^{j-1}}^{(j+1)} & =\hat{b}_{i+2^{j-1}}^{(j)} .
\end{aligned}
$$


Upon the completion of the SC decoding through $\log _{2} N$ levels over $N$ coded bits, Bob will achieve the estimation of all information bits, $\hat{u}_{i}, i \in \mathcal{F}_{c}$.

In addition, Bob may exploit the SCL decoding [27] to further improve the legitimate link performance, by maintaining a list of candidate information bits. Whenever the decoding process arrives at a frozen bit $\hat{u}_{i}, i \in \mathcal{F}$, this bit is added to the list. Whenever an information bit $\hat{u}_{i}, i \in \mathcal{F}_{c}$, is encountered, the bits ' 0 ' and ' 1 ' are respectively added to two replicas of the list, forming two candidate decoding paths. The total number of candidate decoding paths maintained in the list is referred to as the list size, denoted by $T$, which is typically set to a power of two.

2) Eve's Decoding: As is shown in Fig. 1, Eve attempts to detect the information delivered in the legitimate link, based on her received signals $\mathbf{y}_{\mathrm{E}}$ given by (2).

To begin with, the LLR of $v_{i}$ is calculated by Eve as

$$
L L R_{\mathrm{E}}\left(v_{i}\right)=\ln \frac{\sum_{v_{i}=0} \exp \left(-\frac{\left|y_{\mathrm{E}}\left(v_{i}\right)-g s\left(v_{i}\right)\right|^{2}}{\sigma_{Z}^{2}}\right)}{\sum_{v_{i}=1} \exp \left(-\frac{\left|y_{\mathrm{E}}\left(v_{i}\right)-g s\left(v_{i}\right)\right|^{2}}{\sigma_{Z}^{2}}\right)},
$$

where $y_{\mathrm{E}}\left(v_{i}\right)$ stands for Eve's received signal that contains the bit $v_{i}, i=1,2, \cdots, N$.

Since Eve knows the polar encoding matrix $\mathbf{E}_{N}$, she will be able to adopt the SC decoding illustrated in Fig. 2 as well, for the estimation of Alice's transmitted bits $u_{i}, i \in \mathcal{F} \cup \mathcal{F}_{c}$. The LLRs at the start of Eve's SC decoding process are set to those obtained by (12), i.e., $\eta_{i}^{\left(\log _{2} N+1\right)}=L L R_{\mathrm{E}}\left(v_{i}\right), i=$ $1,2, \cdots, N$. Furthermore, Eve may exploit the SCL decoding as well, through maintaining a list of $T$ candidate decoding paths.

The main difference between Eve's and Bob's decoding lies in the reality that Eve does not know the pattern of frozen bits engaged in Alice's polar coding. Therefore, Eve has to estimate all the bits $b_{i}^{(j)}$ based on their LLR values, as given by (8), $\forall i \in\{1,2, \cdots, N\}, j \in\left\{1,2, \cdots, \log _{2} N\right\}$.

\section{Security Performance}

The security performance of our proposed SOPC via channel-gain-mapped frozen bits is evaluated through comparing Bob's and Eve's error rates. To investigate the PLS guaranteed by the SOPC design in the worst-case scenario, we always assume that Eve has the most powerful wiretapping capability and perfectly knows the wiretapping CSI $g$. Besides, to demonstrate the full benefits of our SOPC in the PLS, the legitimate CSI $h$ is assumed to be perfectly known by Alice and Bob. As such, Bob perfectly knows the pattern of frozen bits, $\mathbf{u}_{\mathcal{F}}^{(p)}$, which is engaged in Alice's polar coding for each transmission.

With our SOPC design, the entire channel gain range $[0,+\infty)$ is divided into $P=16$ non-overlapping consecutive intervals, according to (3). Based on the reliability calculated using the Bhattacharyya parameter, the number of information bits, $K_{p}$, pertaining to the interval $\left[\alpha_{p-1}, \alpha_{p}\right)$, $p=1,2, \cdots, 16$, is listed in Table $\mathbf{I}$, where the Bhattacharyya
TABLE I

Security-Oriented Polar Code Design for $P=16$.

\begin{tabular}{clrrrr}
\hline \multirow{2}{*}{ Channel Gain } & \multicolumn{2}{c}{$K_{p}(N=64)$} & \multicolumn{2}{c}{$K_{p}(N=128)$} \\
& Interval & $B_{\mathrm{th}}=0.1$ & 0.2 & $B_{\mathrm{th}}=0.1$ & 0.2 \\
\hline 1 & {$[0,0.0645)$} & 0 & 0 & 0 & 0 \\
2 & {$[0.0645,0.1335)$} & 1 & 3 & 4 & 5 \\
3 & {$[0.1335,0.2076)$} & 4 & 6 & 7 & 12 \\
4 & {$[0.2076,0.2877)$} & 5 & 9 & 13 & 18 \\
5 & {$[0.2877,0.3747)$} & 9 & 12 & 17 & 24 \\
6 & {$[0.3747,0.4700)$} & 11 & 15 & 24 & 31 \\
7 & {$[0.4700,0.5754)$} & 14 & 19 & 29 & 37 \\
8 & {$[0.5754,0.6931)$} & 17 & 22 & 36 & 43 \\
9 & {$[0.6931,0.8267)$} & 20 & 26 & 41 & 51 \\
10 & {$[0.8267,0.9808)$} & 22 & 30 & 48 & 57 \\
11 & {$[0.9808,1.1632)$} & 26 & 33 & 54 & 64 \\
12 & {$[1.1632,1.3863)$} & 31 & 37 & 62 & 72 \\
13 & {$[1.3863,1.6740)$} & 34 & 41 & 71 & 80 \\
14 & {$[1.6740,2.0794)$} & 39 & 45 & 80 & 89 \\
15 & {$[2.0794,2.7726)$} & 44 & 49 & 89 & 98 \\
16 & {$[2.7726,+\infty)$} & 51 & 55 & 102 & 109 \\
\hline
\end{tabular}

parameter threshold is denoted by $B_{\mathrm{th}}$, i.e., the bit-channels having a Bhattacharyya parameter less than $B_{\mathrm{th}}$ are utilised to convey the information bits. Herein, the polar codes of length $N=64$ and $N=128$ are taken into account. With $N=64$, the average code rate, i.e., $(1 / P) \sum_{p=1}^{P} K_{p} / N$, is 0.3203 for $B_{\text {th }}=0.1$ and 0.3926 for $B_{\text {th }}=0.2$. With $N=128$, the average code rate is 0.3306 for $B_{\mathrm{th}}=0.1$ and 0.3857 for $B_{\mathrm{th}}=0.2$.

The bit error rate (BER) and the block error rate (BLER) are compared between the legitimate receiver Bob and the eavesdropper Eve in Figs. 3(a) and 3(b), respectively, where Alice adopts binary phase shift keying (BPSK) modulation, i.e., the energy per bit $E_{b}=E_{s}$. The SOPC has the polar code length $N=64$, and the number of information bits, $K_{p}$, in each channel gain interval is listed in Table I. Bob and Eve exploit the SC and SCL decoders. The list size is set to $T=8$ in their SCL decoding. Fig. 3 reveals that a BLER is $N$ times the corresponding BER in the same condition, which aligns with the intuition. In the case of $B_{\mathrm{th}}=0.1$, our SOPC design degrades Eve's performance by over $12 \mathrm{~dB}$, compared with Bob's performance. As anticipated, a higher threshold $B_{\text {th }}$ results in a lower reliability and a higher polar code rate, thereby leading to worse performance of both Bob and Eve. With the increase in $B_{\mathrm{th}}$, the performance gap between Bob and Eve gets slightly smaller.

Moreover, Bob's performance is improved by utilising the SCL decoding. By contrast, since Eve cannot ascertain the legitimate channel gain $|h|^{2}$, she is unable to identify the frozen bit pattern $\mathbf{u}_{\mathcal{F}}^{(p)}$ engaged in Alice's polar coding and, therefore, her performance cannot be improved by maintaining a list of candidate decoding paths. The main reason behind this is that, without taking the correct frozen bit pattern as a 


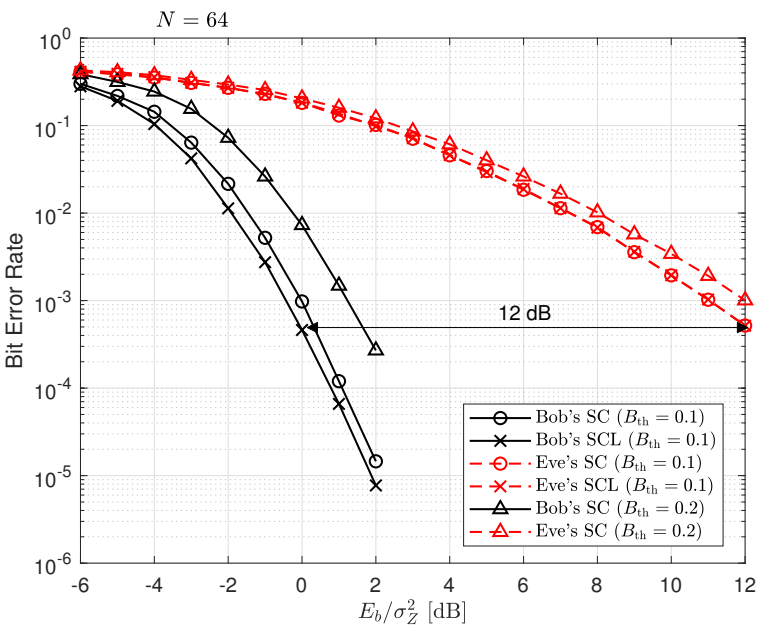

(a) BER

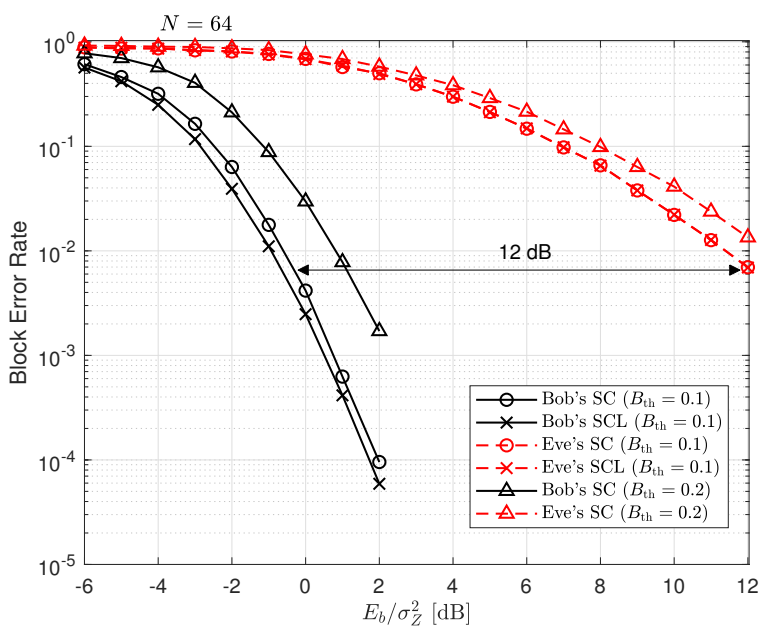

(b) BLER

Fig. 3. BER and BLER comparisons between Bob and Eve in BPSKmodulated systems employing the SOPC with $N=64$.

roadmap, the SCL decoder cannot guarantee the correctness of pruned paths.

Fig. 4 further investigates the PLS functioning of our SOPC design with a longer polar code length, i.e., $N=128$, using the same settings as of Fig. 3. The number of information bits in each channel gain interval, $K_{p}$, is listed in the last two columns of Table I, for the cases of $B_{\text {th }}=0.1$ and 0.2 . By comparing Figs. 3 and 4, we may find that Bob's performance gets better while Eve's performance gets worse, as the polar code length $N$ increases. It can be observed from Fig. 4 that the SOPC design with $N=128$ degrades the eavesdropper's performance by over $13 \mathrm{~dB}$ in comparison with the desired receiver's performance. As the threshold $B_{\text {th }}$ increases, the performance of both Bob and Eve gets worse, and the performance gap between them gets slightly smaller. Furthermore, the SCL decoding improves Bob's performance, but cannot help with Eve's performance at all. A similar phenomenon has been observed in Fig. 3 as well.

Next, the impact of the number of channel gain intervals, $P$, on the PLS functioning of our SOPC design is investigated

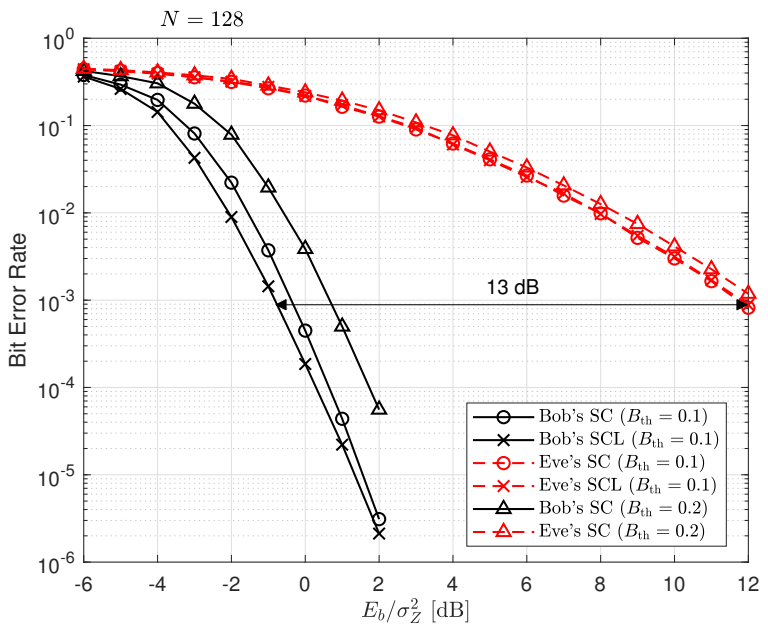

(a) BER

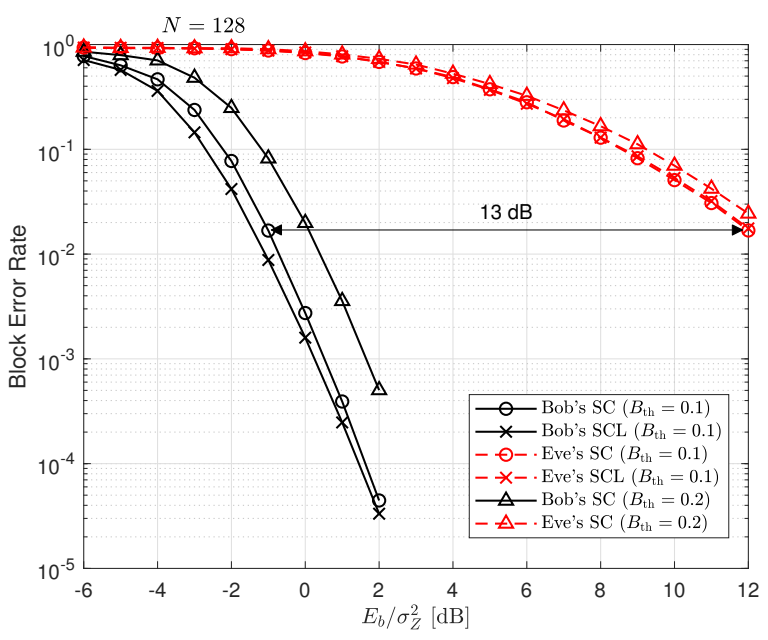

(b) BLER

Fig. 4. BER and BLER comparisons between Bob and Eve in BPSKmodulated systems employing the SOPC with $N=128$.

in Fig. 5, where the BLER of $P=16$ is compared with that of $P=10$ in BPSK-modulated systems using the polar code length $N=64$ and the Bhattacharyya parameter threshold $B_{\mathrm{th}}=0.1$. The settings for $P=16$ and $P=10$ are detailed in Tables I and II, respectively. As shown in Fig. 5, Bob using $P=10$ intervals achieves slightly lower BLER than using $P=16$ at low SNRs. As the SNR increases, Bob's BLER is almost the same in the cases of $P=10$ and $P=16$. The main reason behind this is that the average code rate using $P=10$ is 0.3078 , slightly lower than that using $P=16$, which is 0.3203 . Elaborating slightly further, comparing the number of information bits, $K_{p}$, between Tables I and II, we may find that, given the same channel gain $|h|^{2}$, the polar code rate $K_{p} / N$ with $P=16$ is higher than that with $P=10$.

When it comes to Eve, her BLER is almost the same in the cases of $P=10$ and $P=16$ at low SNRs. As the SNR increases, she achieves slightly lower BLER in the case of $P=$ 10 than in the case of $P=16$. Actually, Eve's performance is almost not affected by $P$, since she always needs to calculate the LLRs for all the bits transmitted by Alice, i.e., whether 


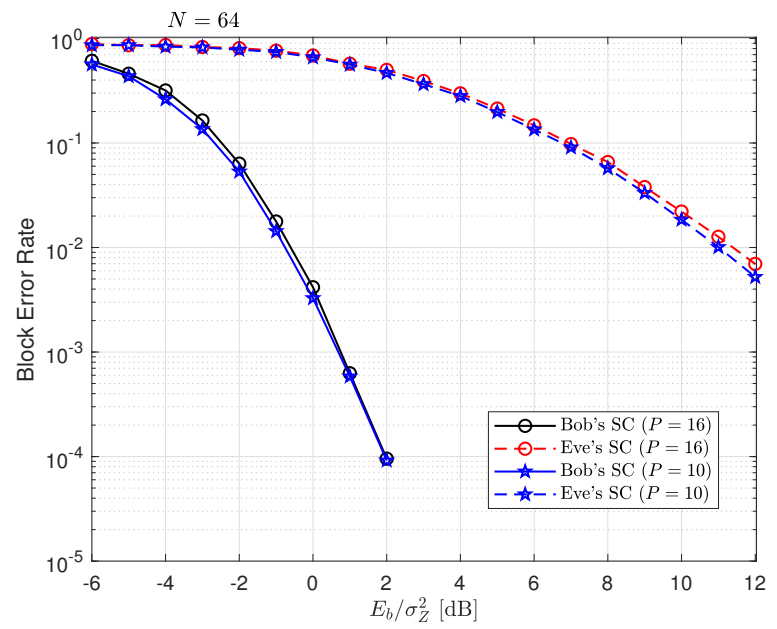

Fig. 5. The impact of the number of channel gain intervals, $P$, on the PLS functioning of our SOPC design in BPSK-modulated systems with $N=64$ and $B_{\mathrm{th}}=0.1$.

TABLE II

Security-Oriented Polar Code Design for $P=10$ and $N=64$ WITH $B_{\text {th }}=0.1$.

\begin{tabular}{rlr}
\hline$p$ & Channel Gain Interval & $K_{p}$ \\
\hline 1 & {$[0,0.1054)$} & 0 \\
2 & {$[0.1054,0.2231)$} & 3 \\
3 & {$[0.2231,0.3567)$} & 6 \\
4 & {$[0.3567,0.5108)$} & 11 \\
5 & {$[0.5108,0.6931)$} & 15 \\
6 & {$[0.6931,0.9163)$} & 20 \\
7 & {$[0.9163,1.2040)$} & 25 \\
8 & {$[1.2040,1.6094)$} & 32 \\
9 & {$[1.6094,2.3026)$} & 38 \\
10 & {$[2.3026,+\infty)$} & 47 \\
\hline
\end{tabular}

the information bits or the frozen bits, in her SC decoding, based on the uniform polar encoding matrix $\mathbf{E}_{N}$. However, Eve has no idea on the location or the number of information bits in a block, as she does not know the active frozen bit pattern. We remark that, Eve's BLER is counted from a genie's perspective, where the information bits are fetched anyway for the comparison purpose. As such, Eve's BLER counted by the genie is reduced by the case of $P=10$, mainly because the number of information bits in this case is less than that in the case of $P=16$. Thanks to the adaptive code rate in polar coding, the number of channel gain intervals has negligible influence on the BLER gap between Bob and Eve, given that Eve carries out the decoding presented in Section II-C-2).

In addition, if Eve is located in very close proximity to Bob, her channel coefficients will be spatially correlated with Bob's. The spatially correlated channels are expressed by the most general correlation model as

$$
\left[\begin{array}{ll}
h_{\mathrm{sc}} & g_{\mathrm{sc}}
\end{array}\right]=[h g] \mathbf{R}^{1 / 2},
$$

where $h_{\mathrm{sc}}$ and $g_{\mathrm{sc}}$ are Bob's and Eve's channels of spatial

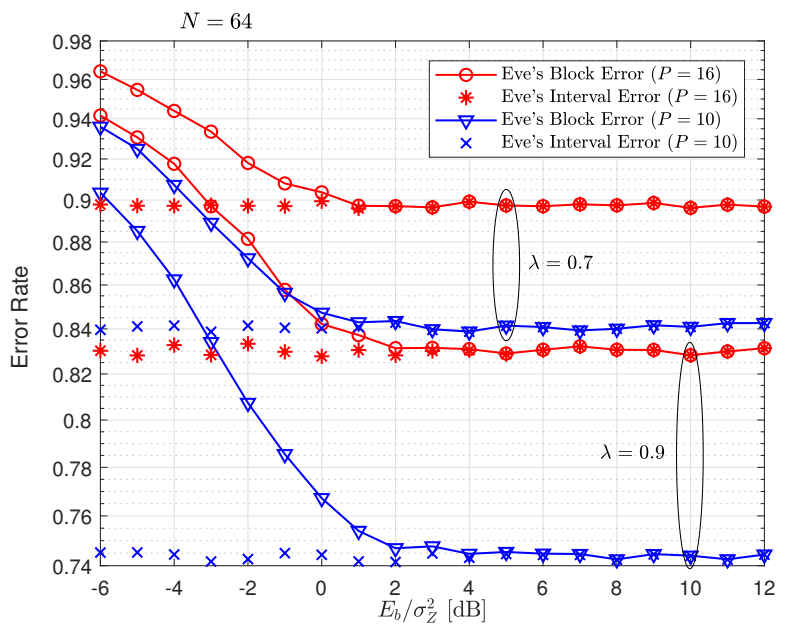

Fig. 6. Eve's SC performance using anticipated frozen bit pattern, subject to the spatial correlation factor between Bob and Eve, $\lambda=0.7,0.9$, in BPSKmodulated SOPC systems with $N=64$ and $B_{\text {th }}=0.1$.

correlation. Note that, $h$ and $g$ are independent channels of Bob and Eve. Generally, the correlation matrix is given by a $2 \times 2$ matrix $\mathbf{R}=\left[\begin{array}{cc}1 & \lambda \\ \lambda^{*} & 1\end{array}\right]$ with a complex correlation factor $\lambda$. Herein, the correlation factor $\lambda$ between Bob and Eve is real [30], and thus $\mathbf{R}=\left[\begin{array}{ll}1 & \lambda \\ \lambda & 1\end{array}\right]$.

To take advantage of the spatial correlation with Bob, Eve is assumed to know the partition of channel gain in (3) and the design of frozen bits in (4), through which Alice formats the SOPC based on the legitimate channel gain $\left|h_{\mathrm{sc}}\right|^{2}$. Then, Eve will determine the active pattern of frozen bits based on her own channel gain $\left|g_{\mathrm{sc}}\right|^{2}$ and carry out the decoding in the same way as Bob does, i.e., the decoding presented in Section II-C-1) instead of II-C-2). Using her anticipated frozen bit pattern for the recovery of Alice's information, Eve's SC performance is investigated in Fig. 6, where the spatial correlation factor between Bob and Eve, $\lambda$, is set to 0.7 and 0.9 . Alice adopts BPSK and SOPC with $N=64, B_{\mathrm{th}}=0.1$. The system settings are detailed in Tables I and II for $P=16$ and $P=10$, respectively. As shown in this figure, although the spatial correlation between Bob and Eve is very high, even to $\lambda=0.9$, Eve still cannot rely on her own channel gain to correctly anticipate the active frozen bit pattern in the legitimate link, because a slight difference between Bob's and Eve's channel gains results in different frozen bit patterns. Moreover, a wrong anticipation of the frozen bit pattern causes the information bit length mismatch in Eve's decoding, as different frozen bit patterns have different lengths, i.e., the $p^{\text {th }}$ pattern $\mathbf{u}_{\mathcal{F}}^{(p)}$ contains $N-K_{p}$ frozen bits. Apparently, Eve's BLER converges to the error rate of her channel gain intervals. With the increase in the number of channel gain intervals, $P$, the chance that Eve's anticipated frozen bit patterns are different from those activated in the legitimate link gets higher. This explains why Eve's performance gets worse in the case of more channel gain intervals involved, given that she relies on the spatial correlation and carries out simple decoding based on her own channel gain.

In summary, our SOPC design with more channel gain 


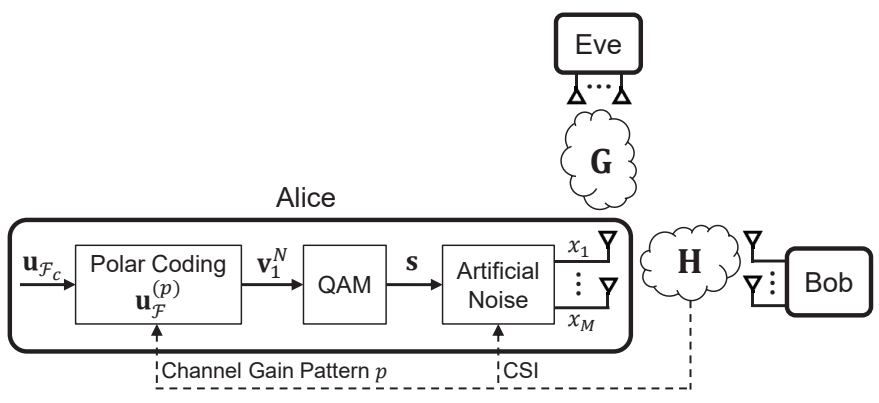

Fig. 7. The SOPC in a MIMOME channel.

intervals achieves slightly better adaptivity but slightly lower security, since both the average code rate of the legitimate link and the performance of the wiretapping link are slightly improved. In case these negligible improvements are taken into account, the tradeoff between adaptivity and security is a concern when choosing the number of channel gain intervals in the SOPC design.

\section{SOPC IN MIMOME NETWORKS}

In this section, the SOPC design is embraced by the artificial noise aided jamming to improve the PLS of MIMOME networks.

\section{A. Network Model}

Consider the application of our SOPC design in a MIMOME network shown in Fig. 7, where Alice has $M$ TAs and Bob has $N_{\mathrm{B}}$ receive antennas (RAs). For the artificial noise injection, we have $M>N_{\mathrm{B}}$. Eve has $N_{\mathrm{E}}$ RAs. The flat-fading channels spanning from Alice to Bob and Eve are denoted by an $N_{\mathrm{B}} \times M$ matrix $\mathbf{H}=\left[h_{n m}\right]_{N_{\mathrm{B}} \times M}=\left[\mathbf{h}_{1}, \mathbf{h}_{2}, \cdots, \mathbf{h}_{M}\right]$ and an $N_{\mathrm{E}} \times M$ matrix $\mathbf{G}=\left[g_{n m}\right]_{N_{\mathrm{E}} \times M}=\left[\mathbf{g}_{1}, \mathbf{g}_{2}, \cdots, \mathbf{g}_{M}\right]$, respectively, where the $N_{\mathrm{B}} \times 1$ vector $\mathbf{h}_{m}=\left[h_{1 m}, h_{2 m}, \cdots, h_{N_{\mathrm{B}} m}\right]^{\mathrm{T}}$ contains the channel coefficients from the $m^{\text {th }}$ TA of Alice to all RAs of $\mathrm{Bob}$, and the $N_{\mathrm{E}} \times 1$ vector $\mathbf{g}_{m}=$ $\left[g_{1 m}, g_{2 m}, \cdots, g_{N_{\mathrm{E}} m}\right]^{\mathrm{T}}$ contains the channel coefficients from the $m^{\text {th }}$ TA of Alice to all RAs of Eve, $m=1,2, \cdots, M$, $n=1,2, \cdots, N_{\mathrm{B}}, N_{\mathrm{E}}$.

The legitimate and wiretapping links are i.i.d. block Rayleigh-fading channels of block length $L$. Given a channel realisation, the coefficients $h_{n m}$ and $g_{n m}$ are i.i.d. complex Gaussian random variables with zero-mean and unit variance. The $N_{\mathrm{B}} \times 1$ vector $\mathbf{h}_{m} \sim \mathcal{C N}\left(\mathbf{0}_{N_{\mathrm{B}} \times 1}, \mathbf{I}_{N_{\mathrm{B}}}\right)$ and the $N_{\mathrm{E}} \times 1$ vector $\mathbf{g}_{m} \sim \mathcal{C N}\left(\mathbf{0}_{N_{\mathrm{E}} \times 1}, \mathbf{I}_{N_{\mathrm{E}}}\right)$.

To better guarantee the PLS of legitimate link, Alice injects artificial noise into the null space of $\mathbf{H}$ to degrade Eve's wiretapping performance without interfering Bob. In an arbitrary channel use, Alice's transmitted signals can be expressed by an $M \times 1$ vector as

$$
\mathbf{x}=\sqrt{\beta E_{s}}\left[\begin{array}{c}
\mathbf{s} \\
\mathbf{0}_{N_{\mathrm{A}}}
\end{array}\right]+\sqrt{(1-\beta) E_{s} \frac{N_{\mathrm{B}}}{N_{\mathrm{A}}}} \mathbf{A} \mathbf{z}_{\mathrm{A}},
$$

where the fraction $\beta \in(0,1)$ is used to adjust the power allocation between the information-bearing QAM symbols $\mathbf{s} \in$ $\mathbb{C}^{N_{\mathrm{B}} \times 1}$ and the artificial noise $\mathbf{z}_{\mathrm{A}} \in \mathbb{C}^{N_{\mathrm{A}} \times 1}$. More specifically, the ratio of the power allocated on the vector $\mathbf{s}$ to the power allocated on the vector $\mathbf{z}_{\mathrm{A}}$ is $\beta /(1-\beta)$. The number of entries in the vector $\mathbf{z}_{\mathrm{A}}$, denoted by $N_{\mathrm{A}}$, is equal to the number of dimensions in the null space of $\mathbf{H}$. In general, $N_{\mathrm{A}}=M-N_{\mathrm{B}}$. The weighting matrix for the artificial noise, $\mathbf{A} \in \mathbb{C}^{M \times N_{\mathrm{A}}}$, lies in the null space of $\mathbf{H}$ with mutually orthogonal columns, and the artificial noise vector is set to $\mathbf{z}_{\mathrm{A}} \sim \mathcal{C N}\left(\mathbf{0}_{N_{\mathrm{A}} \times 1}, \mathbf{I}_{N_{\mathrm{A}}}\right)$ with $N_{\mathrm{A}}$ i.i.d. entries.

As such, the received signals at Bob and Eve are obtained by

$$
\mathbf{y}_{\mathrm{B}}=\mathbf{H} \mathbf{x}+\mathbf{z}_{\mathrm{B}}=\sqrt{\beta E_{s}}\left[\mathbf{h}_{1} \cdots \mathbf{h}_{N_{\mathrm{B}}}\right] \mathbf{s}+\mathbf{z}_{\mathrm{B}}
$$

and

$$
\begin{aligned}
\mathbf{y}_{\mathrm{E}} & =\mathbf{G} \mathbf{x}+\mathbf{z}_{\mathrm{E}} \\
& =\sqrt{\beta E_{s}} \mathbf{G}\left[\begin{array}{c}
\mathbf{s} \\
\mathbf{0}_{N_{\mathrm{A}}}
\end{array}\right]+\sqrt{(1-\beta) E_{s} \frac{N_{\mathrm{B}}}{N_{\mathrm{A}}}} \mathbf{G} \mathbf{A} \mathbf{z}_{\mathrm{A}}+\mathbf{z}_{\mathrm{E}},
\end{aligned}
$$

respectively, where the $N_{\mathrm{B}} \times 1$ vectors $\mathbf{y}_{\mathrm{B}}$ and $\mathbf{z}_{\mathrm{B}} \sim$ $\mathcal{C N}\left(\mathbf{0}_{N_{\mathrm{B}} \times 1}, \sigma_{Z}^{2} \mathbf{I}_{N_{\mathrm{B}}}\right)$ contain Bob's received signals and AWGN components, respectively. The $N_{\mathrm{E}} \times 1$ vectors $\mathbf{y}_{\mathrm{E}}$ and $\mathbf{z}_{\mathrm{E}} \sim \mathcal{C N}\left(\mathbf{0}_{N_{\mathrm{E}} \times 1}, \sigma_{Z}^{2} \mathbf{I}_{N_{\mathrm{E}}}\right)$ contain Eve's received signals and AWGN components, respectively.

\section{B. Polar Code Design}

Different from the polar code design introduced in Subsection II-B for single-antenna wiretap channels, the frozen bit pattern engaged in the SOPC at Alice for MIMOME networks is determined by the instantaneous channel gain pattern of the legitimate channel $\mathbf{H}$. This is equivalent to generating a physical-layer secret key via the channel gain pattern. Thanks to the channel reciprocity of the TDD mode, no feedback channel is needed to deliver the channel coefficients. Therefore, only Alice and Bob know the channel gain pattern as well as the frozen bit pattern of each channel realisation. Eve has no information on the physical-layer secret key; thus her wiretapping performance will be degraded, since she does not have the correct frozen bit pattern for the polar decoding.

In the legitimate link, the channel gain from the $m^{\text {th }} \mathrm{TA}$ of Alice to all RAs of Bob is denoted by $\gamma_{m}=\mathbf{h}_{m}^{\dagger} \mathbf{h}_{m}$. The channel gain pattern is defined as the permutation containing all $\gamma_{m}$, i.e., for $m=1,2, \cdots, M$, in descending or ascending order. For example, in the case with $M=2$, there are two channel gain patterns in descending order: Pattern 1 is $\gamma_{1}>\gamma_{2}$ and Pattern 2 is $\gamma_{2}>\gamma_{1}$. In the case with $M=4$, there are $4 !=24$ permutations of the 4 channel gains, including $\gamma_{1}>\gamma_{2}>\gamma_{3}>\gamma_{4}, \gamma_{4}>\gamma_{3}>\gamma_{2}>\gamma_{1}$, and so on. From an ergodic view point, the total number of channel gain patterns in the legitimate link is $P=M !$ and these patterns occur at the same probability.

For the SOPC design in a MIMOME network, the $P=M$ ! candidate patterns of frozen bits are denoted by $\mathbf{u}_{\mathcal{F}}^{(1)}, \mathbf{u}_{\mathcal{F}}^{(2)}, \cdots, \mathbf{u}_{\mathcal{F}}^{(P)}$. All these $P$ candidate patterns contain the same number of frozen bits, $N-K$, and the polar code rate $K / N$ is stipulated to achieve the equivalent capacity of 
the legitimate channel realisation $\mathbf{H}$, i.e.,

$$
\log _{2}\left(1+\frac{\beta E_{s}}{\sigma_{Z}^{2} N_{\mathrm{B}}} \sum_{m=1}^{N_{\mathrm{B}}} \gamma_{m}\right) \text { bits/channel use, }
$$

by matching the reliability calculated using the Bhattacharyya parameter [25].

The $p^{\text {th }}$ candidate pattern of frozen bits, $p=1,2, \cdots, P$, can be obtained by

$$
\mathbf{u}_{\mathcal{F}}^{(p)}= \begin{cases}\mathcal{B}(p), & P<2^{N-K} \\ \mathcal{B}\left(p \bmod 2^{N-K}\right), & P \geqslant 2^{N-K}\end{cases}
$$

For an arbitrary transmission, if the $p^{\text {th }}$ channel gain pattern occurs in the legitimate link, Alice chooses the $p^{\text {th }}$ pattern of frozen bits, $\mathbf{u}_{\mathcal{F}}^{(p)}$, to encode the $K$ information bits $\mathbf{u}_{\mathcal{F}_{c}}$ into a polar code sequence $\mathbf{v}_{1}^{N}$, as shown in (5).

Given the block fading length $L$ in the legitimate link, the polar coded bits $\mathbf{v}_{1}^{N}$ are modulated by an $A$-ary QAM to generate $L N_{\mathrm{B}}=N / \log _{2} A$ symbols, which is divided into $L$ groups and each group is composed of $N_{\mathrm{B}}$ QAM symbols. Without loss of generality, an arbitrary group of the $N_{\mathrm{B}} \mathrm{QAM}$ symbols is denoted by $\mathbf{s} \in \mathbb{C}^{N_{\mathrm{B}} \times 1}$, which is to be added by the artificial noise $\mathbf{z}_{\mathrm{A}}$, as shown in (14). The generated $M$ signals $\mathbf{x}=\left[x_{1}, x_{2}, \cdots, x_{M}\right]$ are transmitted from Alice's $M$ TAs.

\section{Polar Decoding}

As Bob knows the legitimate channel matrix $\mathbf{H}$ and the frozen bit pattern $\mathbf{u}_{\mathcal{F}}^{(p)}$ engaged in the SOPC at Alice, he can decode the information bits $\mathbf{u}_{\mathcal{F}_{c}}$ using the SC or SCL decoding introduced in Subsection II-C, upon receiving the signals $\mathbf{y}_{\mathrm{B}}$ given by (15). The LLRs at the start of Bob's SC decoding process are calculated using

$$
\begin{aligned}
& L L R_{\mathrm{B}}\left(v_{i}\right) \\
& =\ln \frac{\sum_{v_{i}=0} \exp \left(-\frac{\left\|\mathbf{y}_{\mathrm{B}}\left(v_{i}\right)-\sqrt{\beta E_{s}}\left[\mathbf{h}_{1} \cdots \mathbf{h}_{N_{\mathrm{B}}}\right] \mathbf{s}\left(v_{i}\right)\right\|_{\mathrm{F}}^{2}}{\sigma_{Z}^{2}}\right)}{\sum_{v_{i}=1} \exp \left(-\frac{\left\|\mathbf{y}_{\mathrm{B}}\left(v_{i}\right)-\sqrt{\beta E_{s}}\left[\mathbf{h}_{1} \cdots \mathbf{h}_{N_{\mathrm{B}}}\right] \mathbf{s}\left(v_{i}\right)\right\|_{\mathrm{F}}^{2}}{\sigma_{Z}^{2}}\right)},
\end{aligned}
$$

where $\mathbf{y}_{\mathrm{B}}\left(v_{i}\right)$ and $\mathbf{s}\left(v_{i}\right)$ represent Bob's received signals and Alice's QAM symbols that contain the bit $v_{i}, i=1,2, \cdots, N$.

For the SOPC design in MIMOME networks, Eve's decoding process is the same as that presented in Subsection II-C, based on her received signals $\mathbf{y}_{\mathrm{E}}$ given by (16). For the purpose of investigating the PLS guaranteed by the SOPC in the worst-case scenario, Eve is assumed to know the power $\beta E_{s}$ of an QAM symbol in $\mathbf{s} \in \mathbb{C}^{N_{\mathrm{B}} \times 1}$ through observing her received signals. However, Eve knows neither the legitimate channel matrix $\mathbf{H}$ nor the artificial noise $\mathbf{z}_{\mathrm{A}}$.

At the start of her decoding process, Eve calculates the
LLRs using

$$
\begin{aligned}
& L L R_{\mathrm{E}}\left(v_{i}\right) \\
& =\ln \frac{\sum_{v_{i}=0} \exp \left(-\frac{\left\|\mathbf{y}_{\mathrm{E}}\left(v_{i}\right)-\sqrt{\beta E_{s}}\left[\mathbf{g}_{1} \cdots \mathbf{g}_{N_{\mathrm{B}}}\right] \mathbf{s}\left(v_{i}\right)\right\|_{\mathrm{F}}^{2}}{\sigma_{Z}^{2}}\right)}{\sum_{v_{i}=1} \exp \left(-\frac{\left\|\mathbf{y}_{\mathrm{E}}\left(v_{i}\right)-\sqrt{\beta E_{s}}\left[\mathbf{g}_{1} \cdots \mathbf{g}_{N_{\mathrm{B}}}\right] \mathbf{s}\left(v_{i}\right)\right\|_{\mathrm{F}}^{2}}{\sigma_{Z}^{2}}\right)},
\end{aligned}
$$

where $\mathbf{y}_{\mathrm{E}}\left(v_{i}\right)$ stands for Eve's received signals that contain the bit $v_{i}, i=1,2, \cdots, N$. Then, Eve has to estimate all the bits $b_{i}^{(j)}$ based on their LLR values, as given by (8), $\forall i \in$ $\{1,2, \cdots, N\}, j \in\left\{1,2, \cdots, \log _{2} N\right\}$.

To maximise Eve's wiretapping capability in the interest of evaluating the maximum possible information leakage from our SOPC design, she is assumed to exploit a powerful detector with a brute-force search of all possible frozen bit patterns, $\mathbf{u}_{\mathcal{F}}^{(1)}, \mathbf{u}_{\mathcal{F}}^{(2)}, \cdots, \mathbf{u}_{\mathcal{F}}^{(P)}$, for the recovery of Alice's transmitted information bits $u_{i}, i \in \mathcal{F}_{c}$. We take the polar code $\left(8,4, \mathcal{F}, \mathbf{u}_{\mathcal{F}}^{(p)}\right)$ as an example to elaborate Eve's performance through a brute-force search over all possible frozen bit patterns. Eve's LLRs pertaining to the 16 possible frozen bit patterns used in the SC decoding are shown in Fig. 8, where the frozen bits are located at the indexes $\mathcal{F}=\{1,2,3,5\}$ and the pattern $\mathbf{u}_{\mathcal{F}}^{(p)}=[1,0,1,0]$ is being used by Alice for the polar coding, in a single-antenna system using BPSK modulation.

As shown in Fig. 8(a), when the normalized SNR $E_{b} / \sigma_{Z}^{2}$ is low, e.g., at $0 \mathrm{~dB}$, Eve's LLR values of information bits $u_{i}$, $i \in \mathcal{F}_{c}=\{4,6,7,8\}$, obtained by using the correct frozen bit pattern $[1,0,1,0]$ in the SC decoding occur at random among those obtained through the other frozen bit patterns. In this case, the probability that Eve's extreme LLR values match the correct frozen bit pattern is very low, close to $1 / P$. Thereby, Eve cannot pick up the correct frozen bit pattern at all, even using a brute-force search, because she does not have any basis for the selection.

As shown in Fig. 8(b), when the SNR per bit $E_{b} / \sigma_{Z}^{2}$ is quite high, e.g., at $15 \mathrm{~dB}$, Eve's SC decoding based on the correct frozen bit pattern $[1,0,1,0]$ likely achieves the maximum absolute values of the information bits' LLRs. Therefore, Eve may determine the frozen bit pattern engaged in Alice's polar coding according to the sum over the absolute values of information bits' LLRs. More specifically, the correct frozen bit pattern pertains to the maximum $\sum_{i \in \mathcal{F}_{c}}\left|\eta_{i}^{(1)}\right|$.

\section{Security Performance}

The security performance of our SOPC design in MIMOME channels is evaluated by comparing Bob's and Eve's BERs. Note that, their BLERs are readily obtained by multiplying the corresponding BERs by the polar code length $N$.

To begin with, the worst-case scenario is investigated in Fig. 9, where Alice does not inject artificial noise at all and all the $M$ TAs are fully utilised to deliver the information-bearing symbols $\mathbf{s} \in \mathbb{C}^{N_{\mathrm{B}} \times 1}$, i.e., $\beta=1$ and $M=N_{\mathrm{B}}$. Moreover, Eve exploits a powerful detector through a brute-force search 


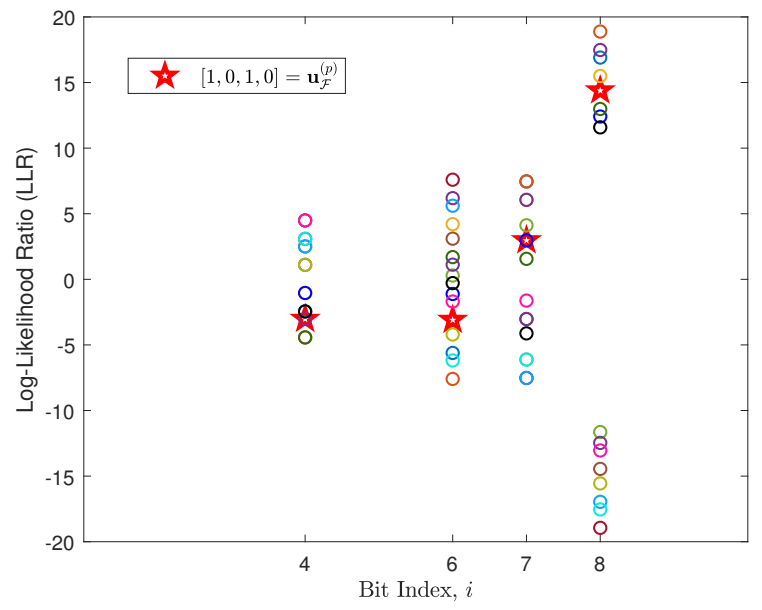

(a) $E_{b} / \sigma_{Z}^{2}=0 \mathrm{~dB}$

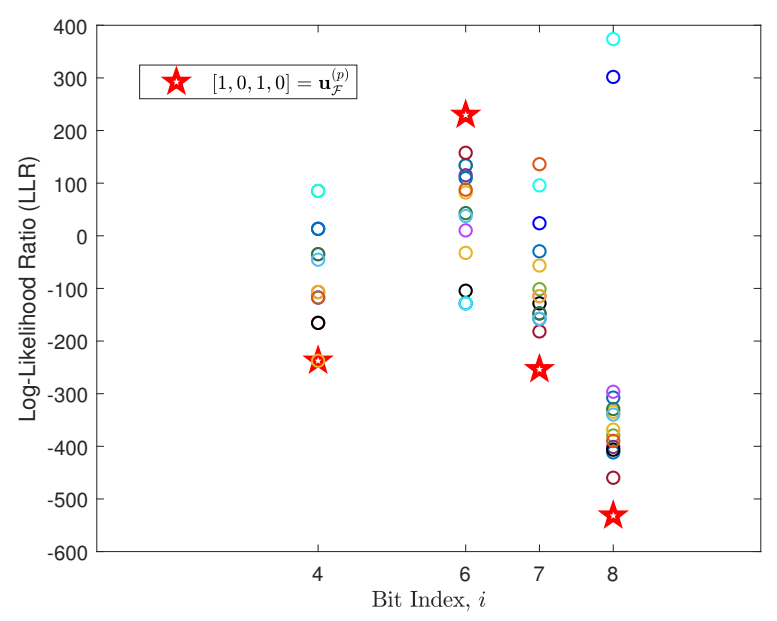

(b) $E_{b} / \sigma_{Z}^{2}=15 \mathrm{~dB}$

Fig. 8. Eve's LLRs pertaining to all 16 possible frozen bit patterns used in the SC decoding, for the polar code $\left(8,4, \mathcal{F}, \mathbf{u}_{\mathcal{F}}^{(p)}\right)$ with $\mathcal{F}=\{1,2,3,5\}$ and $\mathbf{u}_{\mathcal{F}}^{(p)}=[1,0,1,0]$, in a single-antenna system using BPSK modulation.

over all possible frozen bit patterns, and selects the pattern pertaining to the maximum sum over the absolute values of information bits' LLRs for the SC decoding. In the MIMOME network under study, $M=4$ TAs and $N_{\mathrm{B}}=N_{\mathrm{E}}=4$ RAs are set, where the number of legitimate channel gain patterns is $P=M !=24$. Thus, $P=24$ candidate patterns of frozen bits are varied according to the instantaneous channel gain pattern in the legitimate link, and there are $N-K=5$ frozen bits in each pattern. Alice employs BPSK modulation and the fixed-rate polar code $\left(8,3, \mathcal{F}, \mathbf{u}_{\mathcal{F}}^{(p)}\right)$, with $\mathcal{F}=\{1,2,3,4,5\}$ and $\mathbf{u}_{\mathcal{F}}^{(p)}=\mathcal{B}(p), p=1,2, \cdots, 24$.

As shown in Fig. 9, Eve's powerful detector using a bruteforce search slightly improves her decoding performance, and the improvement gets better as the SNR increases. Even so, our SOPC design still degrades Eve's performance by over $5 \mathrm{~dB}$, in comparison to Bob's performance. The main reason behind this phenomenon is that Eve has a high chance to identify the correct frozen bit pattern based on her extreme LLR values

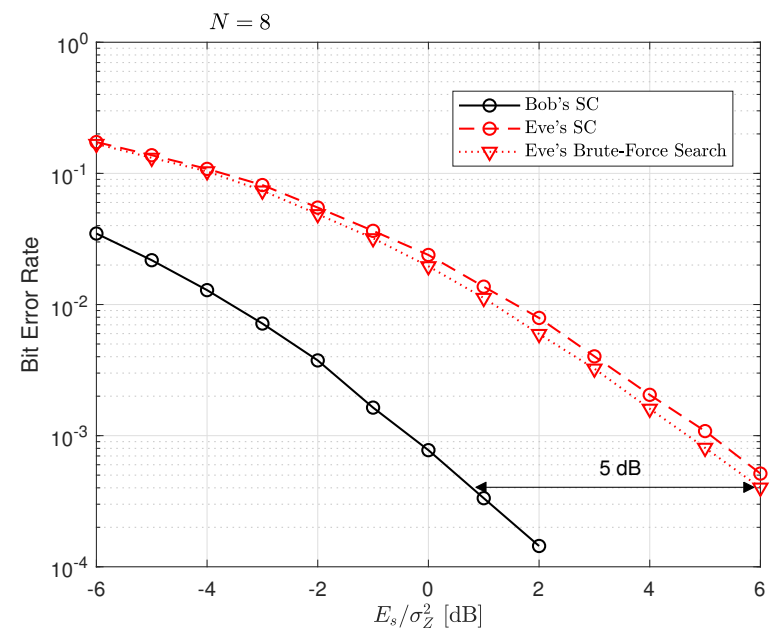

Fig. 9. BER comparisons between Bob ( $\left.N_{\mathrm{B}}=4 \mathrm{RAs}\right)$ and Eve $\left(N_{\mathrm{E}}=4\right.$ RAs) in BPSK-modulated systems employing the SOPC with $\left(8,3, \mathcal{F}, \mathbf{u}_{\mathcal{F}}^{(p)}\right)$ at Alice ( $M=4$ TAs $)$.

when the SNR is very high, e.g., in the case of $E_{b} / \sigma_{Z}^{2}=$ $15 \mathrm{~dB}$ as illustrated by Fig. 8(b). However, at low SNRs, Eve is still unable to obtain the correct frozen bit pattern even using a brute-force search, as shown in Fig. 8(a). As the SNR increases, the probability that Eve identifies the correct frozen bit pattern gets slightly higher.

We remark that, Eve needs to do the brute-force search over $M$ ! possible patterns of frozen bits if she knows how to form the frozen bit pattern for a given channel gain pattern, e.g., (17). If Eve does not know the formulation rule of frozen bit patterns, she needs to carry out the brute-force search over $2^{N-K}$ possible patterns of frozen bits. With the increase in the number of frozen bits, $N-K$, engaged in the legitimate link, unlimited computational resources are required in Eve's decoding with the brute-force search, which, however, results in a very limited performance improvement for Eve.

Further, the PLS enhancement brought by the SOPC with artificial noise injection is exemplified in Fig. 10, where the BER is plotted versus $E_{s} / \sigma_{Z}^{2}$. The energy per symbol, $E_{s}$, is distributed between the information-bearing vector $\mathbf{s}$ and the artificial noise $\mathbf{z}_{\mathrm{A}}$ in the ratio $\beta /(1-\beta)$. Within BPSK-modulated systems, the energy per polar coded bit $E_{b}=\beta E_{s}$. Herein, both Bob and Eve have $N_{\mathrm{B}}=N_{\mathrm{E}}=4$ RAs. For the artificial noise injection, Alice has $M=6$ TAs and the power allocation factor $\beta=0.8$. In (14), the power allocated to an information-bearing BPSK symbol in $\mathbf{s}$ is $0.8 E_{s}$, and the power allocated to an artificial noise component in $\mathbf{z}_{\mathrm{A}}$ is $0.2 E_{s} N_{\mathrm{B}} /\left(M-N_{\mathrm{B}}\right)$. The number of channel gain patterns is $P=6 !=720$. We consider two methods for the construction of the SOPC having code length $N=64$ : code rate adaption and fixed code rate. With the former method, given a ratio $E_{s} / \sigma_{Z}^{2}$, the equivalent channel gain $\left(\beta / N_{\mathrm{B}}\right) \sum_{m=1}^{N_{\mathrm{B}}} \gamma_{m}$ is divided into 16 intervals and the code rate $K / N$ in each interval is determined by the reliability pertaining to the Bhattacharyya parameter threshold $B_{\mathrm{th}}=0$. The average code rate of the SOPC in this method is 0.4004 . With the latter method, the code rate $K / N$ is fixed to $3 / 8$, 


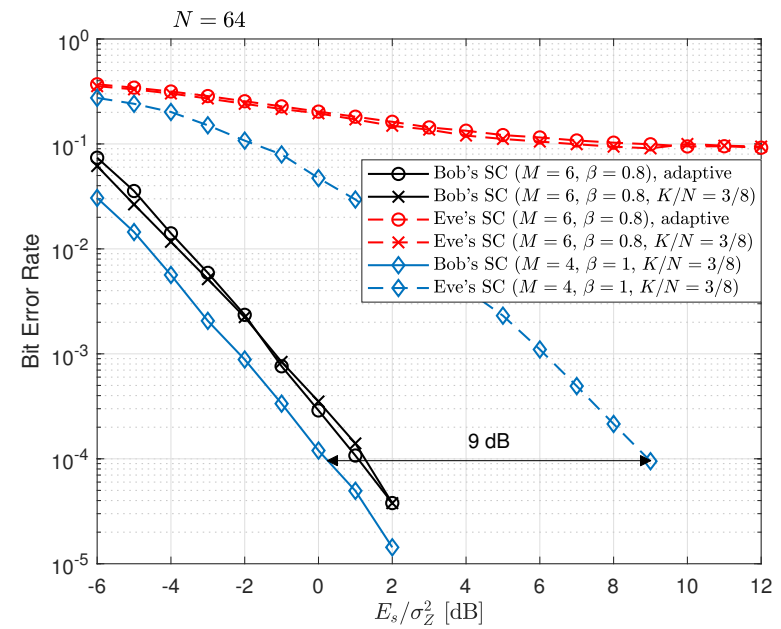

Fig. 10. BER comparisons between Bob $\left(N_{\mathrm{B}}=4 \mathrm{RAs}\right)$ and Eve $\left(N_{\mathrm{E}}=4\right.$ RAs) in BPSK-modulated systems employing the SOPC with $N=64$ at Alice.

i.e., there are always $K=24$ information bits in $\mathbf{u}_{\mathcal{F}_{c}}$. In both methods, the $p^{\text {th }}$ pattern of frozen bits, $\mathbf{u}_{\mathcal{F}}^{(p)}$, can be designed according to (17), $p=1,2, \cdots, 720$. As shown in Fig. 10, Eve's performance for these two methods is almost the same, and so does Bob's performance. This is mainly because of the spatial diversity gain achieved by MIMOME channels. Bob's performance with fixed code rate is slightly better than that with code rate adaption at low SNRs, while slightly worse at high SNRs. The main reason behind this is that the fixed code rate $3 / 8$ is slightly lower than the average code rate in the adaptation, 0.4004, which dominates Bob's performance improvement when the SNR is low. As the SNR increases, the advantage of code rate adaption starts to prevail Bob's performance improvement that results from adaptive bitchannel reliability.

For the purpose of comparison, we also investigate the case of no artificial noise injection in Fig. 10, with $M=4$ TAs at Alice and $\beta=1$, i.e., all the transmit power is allocated to information-bearing BPSK symbols. In this case, the code rate of Alice's SOPC with length $N=64$ is fixed to $3 / 8$ as well. There are 24 channel gain patterns to activate the patterns of frozen bits, $\mathbf{u}_{\mathcal{F}}^{(p)}=\mathcal{B}(p), p=1,2, \cdots, 24$. From the PLS functioning comparison between the SOPC with and without artificial noise, it is observed that the artificial noise injection in MIMOME channels does dramatically enhance the PLS achieved by our SOPC. Without artificial noise injection, our SOPC design leaves a 9dB performance gap between Bob and Eve. When the artificial noise, even allocated with a small fraction $1-\beta=0.2$ of transmit power, is injected in the SOPC, the performance gap between Bob and Eve drastically increases, where Eve's performance gets very poor, with the BER converging to 0.1 . The PLS enhancement not only comes from the artificial noise that degrades Eve's channel quality, but also is attributed to a larger number of channel gain patterns formed by more TAs at Alice.

Moreover, comparing the PLS functioning of SOPC having code length $N=8$ in Fig. 9 to that of $N=64$ without

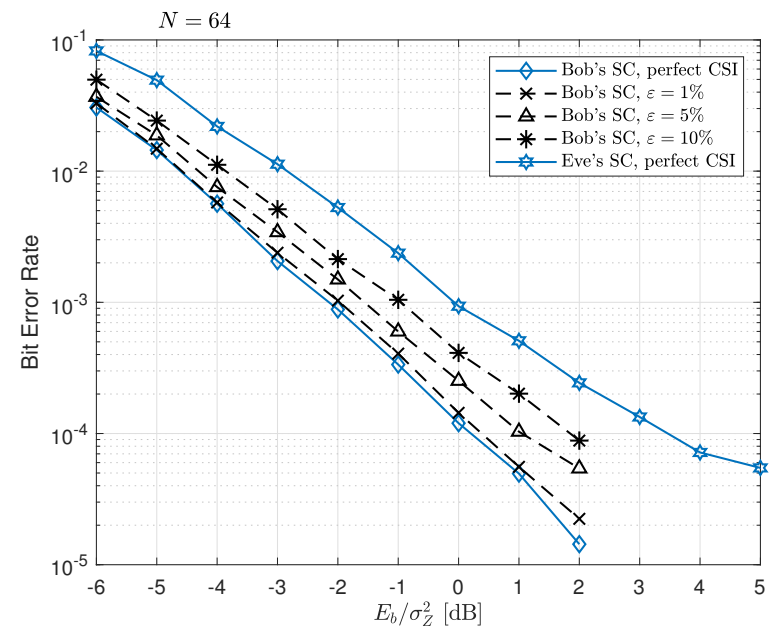

Fig. 11. The impact of CSI estimation error on SOPC performance in BPSKmodulated systems employing $\left(64,24, \mathcal{F}, \mathbf{u}_{\mathcal{F}}^{(p)}\right)$ at Alice, for $M=N_{\mathrm{B}}=$ $N_{\mathrm{E}}=4$.

artificial noise in Fig. 10, we may find that our SOPC with a longer code length exhibits better PLS functioning in MIMOME channels. The performance gap between Bob and Eve is increased from $5 \mathrm{~dB}$ to $9 \mathrm{~dB}$, when the code length of our SOPC is increased from 8 to 64 .

In practice, the time variation of the channel and the error on channel estimation entail that the legitimate CSI is imperfectly known to the transceivers. Using a well known model for the CSI measurement [31], the noisy estimate of the legitimate CSI is modelled as

$$
\hat{\mathbf{H}}=\mathbf{H}+\varepsilon \boldsymbol{\Omega},
$$

where the $N_{\mathrm{B}} \times M$ matrix $\hat{\mathbf{H}}$ contains the estimated channel coefficients. The entries of the $N_{\mathrm{B}} \times M$ matrix $\Omega$ are i.i.d. complex Gaussian random variables with zero-mean and unit variance, and $\varepsilon \boldsymbol{\Omega}$ is the estimation error that is independent of $\mathbf{H}$. The CSI estimation accuracy is measured by $\varepsilon$, and the normalised mean squared error of the CSI estimation is $\varepsilon^{2}$ [32], [33].

The impact of CSI estimation error on Bob's performance is reported in Fig. 11, where Alice adopts BPSK and SOPC with $\left(64,24, \mathcal{F}, \mathbf{u}_{\mathcal{F}}^{(p)}\right)$. To investigate the PLS achieved in the worst-case scenario, Eve is assumed to perfectly know the wiretapping CSI G, and each user is equipped with 4 antennas, i.e., $M=N_{\mathrm{B}}=N_{\mathrm{E}}=4$. More specifically, there is no artificial noise at all.

Herein, the $M !=24$ frozen bit patterns are cautiously designed to guarantee the robustness of legitimate link. As the minimum number of different bits to represent these patterns is $\left\lceil\log _{2} 24\right\rceil=5$, all the other 35 frozen bits are set to the same value, i.e., they are all 0's or 1's. Moreover, the five different bits are located at the indexes $\{1,2,3,5,9\}$, which are the channels of the lowest reliability levels and have the least connections with others.

As is shown in Fig. 11, Bob's performance gets worse with the increase in $\varepsilon$. Comparing this figure with Fig. 10, we may find that Bob maintains the same performance in the case 
perfect CSI, for both the cautious design of frozen bit patterns and the design given by (17). However, Eve's performance is improved by the cautious design, because the influence of incorrectly determining frozen bit patterns is mitigated not only for Bob but also for Eve. Therefore, the cautious design of frozen bit patterns weakens the security provided by the SOPC, although guarantees the legitimate link performance at the presence of CSI estimation error.

To further improve the security, Alice may utilise more TAs to generate artificial noise. In addition, the channel gain from the $m^{\text {th }}$ TA of Alice to Bob, $\gamma_{m}=\mathbf{h}_{m}^{\dagger} \mathbf{h}_{m}$, follows a chi-square distribution with mean $N_{\mathrm{B}}$ and variance $5.5 N_{\mathrm{B}}$. Since the variance of $\gamma_{m}$ linearly increases with the number of Bob's RAs, $N_{\mathrm{B}}$, increasing $N_{\mathrm{B}}$ will reduce the impact of CSI estimation error on the identification of active channel gain pattern, thus boosting Bob's performance. As such, the tradeoff between the security and the cost of multi-antenna configuration has to be taken into account for practical designs.

\section{CONCLUSION}

\section{A. Summary}

In this paper, a novel PLS design, referred to as SOPC, was proposed based on the channel-gain-mapped frozen bits, to degrade Eve's decoding while maintaining Bob's performance within both SISOSE and MIMOME channels. The instantaneous pattern of frozen bits activated by Alice in the SOPC was generated by the legitimate channel gain interval in a SISOSE channel and by the legitimate channel gain pattern in a MIMOME channel, as a physical-layer secret key. Since Eve was blind to the legitimate channel gain, she could not ascertain this secret key, i.e., the frozen bit pattern engaged in Alice's SOPC, and therefore failed to decode the confidential information in an appropriate manner. With the SOPC, Alice did not need to know Eve's CSI, which ensured the flexibility of the PLS implementation. Moreover, we investigated the scenario where Eve might carry out powerful decoding through a brute-force search over all possible patterns of frozen bits. Illustrative simulation results substantiated the validity and robustness of our SOPC design in the PLS enhancement, even facing a powerful eavesdropper possessing infinite computational resources.

\section{B. Discussion}

From the outcome of our PLS functioning evaluations, several important insights were reached to facilitate the SOPCbased system design:

(i) In general, the PLS is improved, i.e., the performance gap between Bob and Eve gets larger, as the polar code length $N$ increases in the SOPC. For example, the gap in SISOSE channels is $12 \mathrm{~dB}$ for $N=64$ and $13 \mathrm{~dB}$ for $N=128$. The gap in MIMOME channels is $5 \mathrm{~dB}$ for $N=8$ and $9 \mathrm{~dB}$ for $N=64$.

(ii) In SISOSE channels, if the legitimate and wiretapping links are i.i.d., the number of channel gain intervals has negligible influence on the PLS achieved by the SOPC, as long as the code rate adaption is exploited. However, the average code rate of SOPC is reduced with the decrease in the number of channel gain intervals.

(iii) In MIMOME channels, the increase in the number of Alice's TAs not only allows the artificial noise injection but also forms more channel gain patterns, for the PLS enhancement. Because of the spatial diversity gain in MIMOME channels, the SOPC with fixed code rate achieves almost the same PLS performance as that with code rate adaption. If Eve carries out a brute-force search over all possible patterns of frozen bits, the SOPC with code rate adaption will boost the computational complexity in Eve's decoding.

\section{Future Work}

The studies we initiated here are just the beginning of our research on the SOPC design. The following features are being pursued to address better performance of the legitimate link while enhancing its security:

(i) As a more advanced polar decoding technique, a cyclic redundancy check code is to be used together with the SCL decoding for improving the legitimate link performance at high SNRs.

(ii) Advanced design of polar coding construction is to be addressed for improving the randomness of secret key in guaranteeing the legitimate link performance, specifically at the presence of CSI estimation error.

(iii) Research on the interactions and combinations of the SOPC design with other techniques, such as one-time pad keys [1] and mapping-varied modulation [20], is to be carried out for further improving the security.

\section{ACKNOWLEDGMENT}

The authors would like to thank the editor, Dr. Vasanthan Raghavan, and the anonymous reviewers for their valuable comments to improve the presentation of this paper.

\section{REFERENCES}

[1] C. E. Shannon, "Communication theory of secrecy systems", Bell Labs Tech. J., vol. 28, no. 4, pp. 656-715, Oct. 1949.

[2] J. Cao, et al., "A survey on security aspects for 3GPP 5G networks", IEEE Commun. Surveys Tuts., vol. 22, no. 1, pp. 170-195, 1st Quart. 2020.

[3] S. Chen, S. Sun, Q. Gao, and X. Su, "Adaptive beamforming in TDDbased mobile communication systems: State of the art and 5G research directions", IEEE Wireless Commun., vol. 23, no. 6, pp. 81-87, Dec. 2016.

[4] J. da Silva, G. Wikstrom, R. Mungara, and C. Fischione, "Full duplex and dynamic TDD: Pushing the limits of spectrum reuse in multi-cell communications", IEEE Wireless Commun., vol. 28, no. 1, pp. 44-50, Feb. 2021.

[5] A. Sayeed and A. Perrig, "Secure wireless communications: Secret keys through multipath", in Proc. IEEE Int. Conf. Acoust. Speech Signal Process. (ICASSP), Apr. 2008, pp. 3013-3016.

[6] IEEE Std 802.16-2017, "IEEE Standard for Air Interface for Broadband Wireless Access Systems", Mar. 2018.

[7] E. Dahlman, S. Parkvall, and J. Skold, 5G NR: The Next Generation Wireless Access Technology. 2nd Ed. USA: Academic Press, 2020.

[8] K. Ren, H. Su, and Q. Wang, "Secret key generation exploiting channel characteristics in wireless communications", IEEE Wireless Commun., vol. 18, no. 4, pp. 6-12, Aug. 2011.

[9] Y. Liu, S. Draper, and A. Sayeed, "Exploiting channel diversity in secret key generation from multipath fading randomness", IEEE Trans. Inf. Forensics Security, vol. 7, no. 5, pp. 1484-1497, Oct. 2012. 
[10] S. Goel and R. Negi, "Guaranteeing secrecy using artificial noise", IEEE Trans. Wireless Commun., vol. 7, no. 6, pp. 2180-2189, Jun. 2008.

[11] J. Wang, J. Lee, F. Wang, and T. Quek, "Jamming-aided secure communication in massive MIMO Rician channels", IEEE Trans. Wireless Commun., vol. 14, no. 12, pp. 6854-6868, Dec. 2015.

[12] Q. Zhang, X. Huang, Q. Li, and J. Qin, "Cooperative jamming aided robust secure transmission for wireless information and power transfer in MISO channels", IEEE Trans. Commun., vol. 63, no. 3, pp. 906-915, Mar. 2015.

[13] K. Zeng, K. Govindan, and P. Mohapatra, "Non-cryptographic authentication and identification in wireless networks", IEEE Wireless Commun., vol. 17 , no. 5, pp. 56-62, Oct. 2010.

[14] X. Duan and X. Wang, "Authentication handover and privacy protection in 5G HetNets using software-defined networking", IEEE Commun. Mag., vol. 53, no. 4, pp. 28-35, Apr. 2015.

[15] Y. Liu, H. Chen, and L. Wang, "Physical layer security for next generation wireless networks: Theories, technologies, and challenges", IEEE Commun. Surveys Tuts., vol. 19, no. 1, pp. 347-376, 1st Quart. 2017.

[16] A. Mukherjee, S. Fakoorian, J. Huang, and A. Swindlehurst, "Principles of physical layer security in multiuser wireless networks: A survey", IEEE Commun. Surveys Tuts., vol. 16, no. 3, pp. 1550-1573, 3rd Quart. 2014.

[17] A. Mukherjee and A. L. Swindlehurst, "Robust beamforming for security in MIMO wiretap channels with imperfect CSI", IEEE Trans. Signal Process., vol. 59, no. 1, pp. 351-361, Jan. 2011.

[18] Y. Yang and B. Jiao, "Artificial-noise strategy for single-antenna systems over multi-path fading channels", in Proc. Int. Wireless Commun. Mobile Comput. Conf. (IWCMC), Aug. 2015, pp. 96-101.

[19] J. M. Hamamreh and H. Arslan, "Joint PHY/MAC layer security design using ARQ with MRC and null-space independent PAPR-aware artificial noise in SISO systems", IEEE Trans. Wireless Commun., vol. 17, no. 9, pp. 6190-6204, Sep. 2018.

[20] Y. Yang and M. Guizani, "Mapping-varied spatial modulation for physical layer security: Transmission strategy and secrecy rate", IEEE J. Sel. Areas Commun., vol. 36, no. 4, pp. 877-889, Apr. 2018.

[21] Y. Yang, M. Ma, S. Aissa, and L. Hanzo, "Physical-layer secret key generation via CQI-mapped spatial modulation in multi-hop wiretap ad- hoc networks", IEEE Trans. Inf. Forensics Security, vol. 16, pp. $1322-$ 1334, 2021.

[22] Y. Liu, Y. Yang, L. Yang, and L. Hanzo, "Physical layer security of spatially modulated sparse-code multiple access in aeronautical ad-hoc networking", IEEE Trans. Veh. Tech., vol. 70, no. 3, pp. 2436-2447, Mar. 2021.

[23] M. Yin, Y. Yang, and B. Jiao, "Security-oriented trellis code design for spatial modulation", IEEE Trans. Wireless Commun., vol. 20, no. 3, pp. 1875-1888, Mar. 2021.

[24] V. Bioglio, C. Condo, and I. Land, "Design of polar codes in 5G new radio", IEEE Commun. Surveys Tuts., vol. 23, no. 1, pp. 29-40, First Quarter 2021.

[25] E. Arikan, "Channel polarization: A method for constructing capacityachieving codes for symmetric binary-input memoryless channels", IEEE Trans. Inf. Theory, vol. 55, no. 7, pp. 3051-3073, Jul. 2009.

[26] C. Leroux, A. Raymond, G. Sarkis, et al., "Hardware implementation of successive-cancellation decoders for polar codes", J. Signal Process. Syst., vol. 69, pp. 305-315, Jul. 2012.

[27] I. Tal and A. Vardy, "List decoding of polar codes", IEEE Trans. Inf. Theory, vol. 61, no. 5, pp. 2213-2226, May 2015.

[28] E. Arikan, "On the origin of polar coding", IEEE J. Sel. Areas Commun., vol. 34, no. 2, pp. 209-223, Feb. 2016

[29] M. Fossorier, M. Mihaljevic, and H. Imai, "Reduced complexity iterative decoding of low-density parity check codes based on belief propagation”, IEEE Trans. Commun., vol. 47, no. 5, pp. 673-680, May 1999.

[30] Y. Yang and S. Aissa, "Information-guided communications in MIMO systems with channel state impairments", Wirel. Commun. Mob. Comput., vol. 15, pp. 868-878, 2015.

[31] M. Medard, "The effect upon channel capacity in wireless communications of perfect and imperfect knowledge of the channel", IEEE Trans. Inf. Theory, vol. 46, no. 3, pp. 933-946, May 2000.

[32] C. Wang, et al., "On the Performance of the MIMO Zero-Forcing Receiver in the Presence of Channel Estimation Error", IEEE Trans. Wireless Commun., vol. 6, no. 3, pp. 805-810, Mar. 2007.

[33] Y. Yang, N. Bonello, and S. Aissa, "An information-guided channelhopping scheme for block-fading channels with estimation errors", in Proc. IEEE Global Commun. Conf. (GLOBECOM), Miami, pp. 1-5, 2010. 